\title{
Mitigation of EC breakdown in the gyrotron transmission line of the ITER Collective Thomson Scattering diagnostic via a Split Biased Waveguide
}

\author{
Larsen, A. W.; Korsholm, S. B.; Gonçalves, B.; Gutierrez, H. E.; Henriques, E.; Infante, V.; Jensen, T.; \\ Jessen, M.; Klinkby, Esben Bryndt; Nonbøl, Erik \\ Total number of authors: \\ 21
}

Published in:

Journal of Instrumentation

Link to article, DOI:

$10.1088 / 1748-0221 / 14 / 11 / c 11009$

Publication date:

2019

Document Version

Peer reviewed version

Link back to DTU Orbit

\section{Citation (APA):}

Larsen, A. W., Korsholm, S. B., Gonçalves, B., Gutierrez, H. E., Henriques, E., Infante, V., Jensen, T., Jessen, M., Klinkby, E. B., Nonbøl, E., Luis, R., Vale, A., Lopes, A., Naulin, V., Nielsen, S. K., Salewski, M., Rasmussen, J., Taormina, A., Møllsøe, C., ... Trieschmann, J. (2019). Mitigation of EC breakdown in the gyrotron transmission line of the ITER Collective Thomson Scattering diagnostic via a Split Biased Waveguide. Journal of Instrumentation, 14(11), [C11009]. https://doi.org/10.1088/1748-0221/14/11/c11009

\section{General rights}

Copyright and moral rights for the publications made accessible in the public portal are retained by the authors and/or other copyright owners and it is a condition of accessing publications that users recognise and abide by the legal requirements associated with these rights.

- Users may download and print one copy of any publication from the public portal for the purpose of private study or research.

- You may not further distribute the material or use it for any profit-making activity or commercial gain

- You may freely distribute the URL identifying the publication in the public portal 


\title{
Mitigation of EC breakdown in the gyrotron transmission line of the ITER Collective Thomson Scattering diagnostic via a Split biased waveguide
}

\author{
A.W. Larsen ${ }^{a}$, S.B. Korsholm ${ }^{a}$, B. Gonçalves ${ }^{b}$, H.E. Gutierrez ${ }^{a}$, E. Henriques ${ }^{d}$, \\ V. Infante ${ }^{d}$, T. Jensen ${ }^{a}$, M. Jessen ${ }^{a}$, E.B. Klinkby ${ }^{c}$, E. Nonbøl', R. Luis ${ }^{b}, A$. Vale ${ }^{b}$, \\ A. Lopes ${ }^{\mathrm{b}}$, V. Naulin ${ }^{\mathrm{a}}$, S.K. Nielsen ${ }^{\mathrm{a}}$, M. Salewski ${ }^{\mathrm{a}}$, J. Rasmussen ${ }^{\mathrm{a}}$, A. Taormina ${ }^{\mathrm{a}}$, \\ C. Møllsøe ${ }^{\mathrm{a}}, \mathrm{T}$. Mussenbrock ${ }^{\mathrm{e}}, \mathrm{J}$. Trieschmann ${ }^{\mathrm{e}}$. \\ ${ }^{a}$ Technical University of Denmark, Department of Physics, 2800 Kgs. Lyngby, Denmark \\ ${ }^{b}$ Instituto de Plasmas e Fusão Nuclear, Instituto Superior Técnico, Univ. Lisboa, Portugal \\ ${ }^{c}$ Technical University of Denmark, Center for Nuclear Technologies, 4000 Roskilde, Denmark \\ ${ }^{d}$ IDMEC, Instituto Superior Técnico, Univ. Lisboa, Portugal \\ ${ }^{e}$ Brandenburg University of Technology Cottbus-Senftenberg, Cottbus, Germany \\ E-mail: axla@fysik.dtu.dk
}

ABSTRACT: In this paper we present the results of the R\&D work that has been performed on avoiding electron cyclotron (EC) gas breakdown inside the launcher transmission line (TL) of the ITER collective Thomson scattering (CTS) diagnostic, due to encountering the fundamental EC resonance, which is located inside the port plug vacuum for the baseline ITER magnetic field scenario. If an EC breakdown occurs, this can lead to strong local absorption of the CTS gyrotron beam, as well as arcing inside the ITER vacuum vessel, which must be avoided. Due to the hostile, restrictive, and nuclear environment in ITER, it is not possible to implement the standard method for avoiding EC breakdown - a controlled atmosphere at the EC resonance. Instead, the CTS diagnostic will include a longitudinally-split electrically-biased corrugated waveguide (SBWG) in the launcher transmission line. The SBWG works by applying a transverse DC bias voltage across the two electrically-isolated waveguide halves, causing free electrons to diffuse out of the EC resonant region before they can cause an electron-impact ionisation-avalanche, and thus an EC breakdown. Due to insufficient experimental facilities, the functionality of the SBWG is validated through Monte Carlo electron modelling.

KEYWORDS: ITER; CTS; EC Breakdown; Fusion; Gyrotron. 


\section{Introduction}

The ITER Collective Thomson Scattering (CTS) diagnostic, which is located within diagnostic shielding module (DSM) \#3 of equatorial port plug \#12 (EPP12), will diagnose the velocity distribution of the fusion-born alpha particles $\left(\$ 3.5 \mathrm{MeV}{ }^{4} \mathrm{He}\right)$ in ITER, by observing the scattering of a high-power microwave beam (1 MW@60 GHz) from seven individual scattering volumes, located at different radial positions inside the ITER plasma (see Figure 1) [1]. The CTS can detect alpha particles at energies down to $\sim 0.3 \mathrm{MeV}$, and is the only ITER diagnostic, which will be able to diagnose the fusion-born alphas with energies $<1.7 \mathrm{MeV}$ [2].

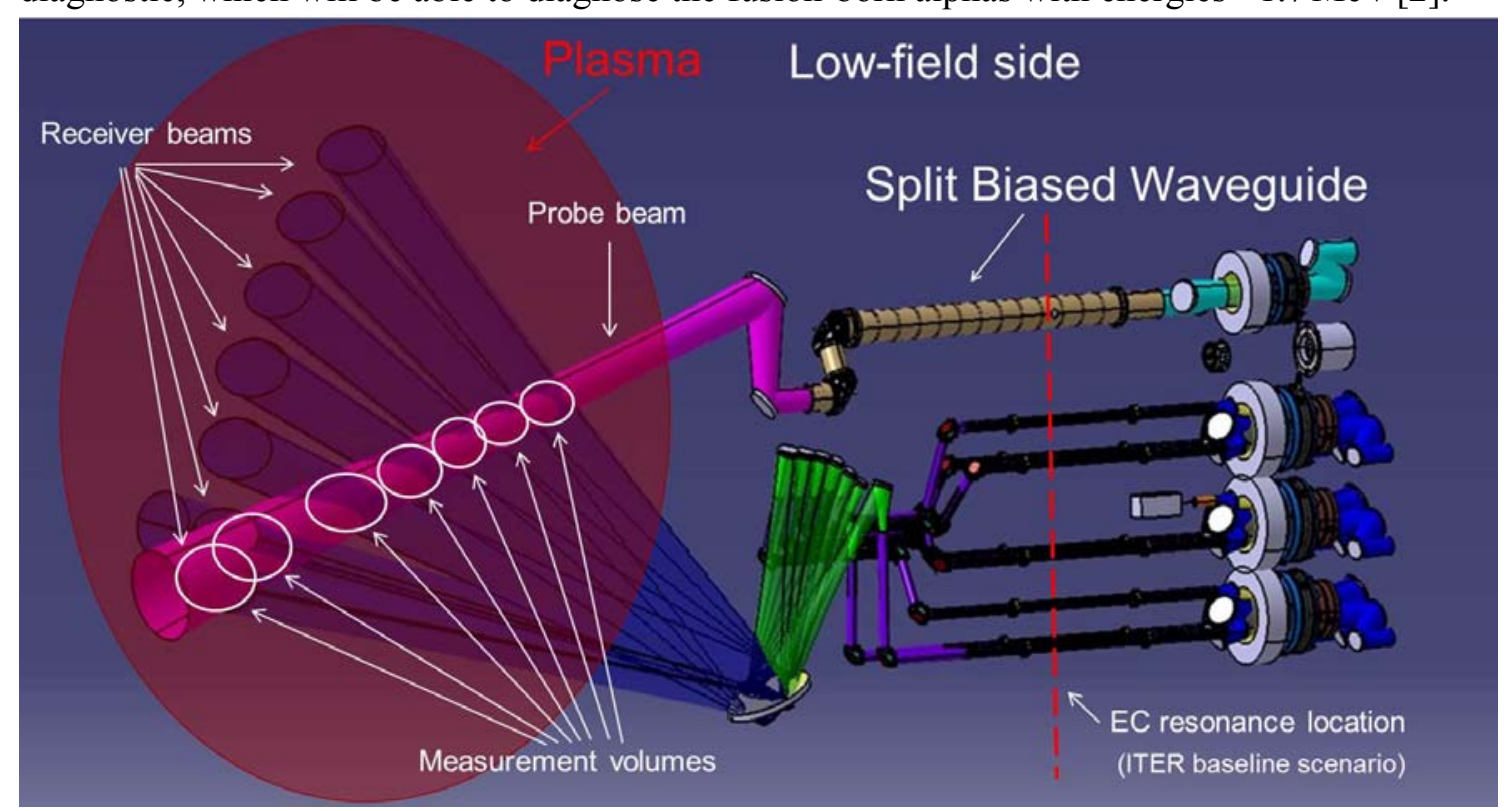

Figure 1: CAD outline of the front-end of the ITER CTS diagnostic system. The top transmission line is the one carrying the pink $1 \mathrm{MW}$ gyrotron beam, with the SBWG marked by an arrow. The approximate location of the EC resonance (in the ITER baseline scenario) is marked by a dashed red vertical line.

Due to the estimated level of microwave background radiation in the ITER plasma [3], the best diagnostic performance is achieved for a probing frequency of $60 \mathrm{GHz}$ [4]. For the ITER baseline scenario (see section 1.2), this means that the fundamental EC resonance will be encountered inside EPP12, where the CTS front-end components are located. The high-power probe beam will interact with the EC resonance, and if the gas pressure rises too high in the TL (e.g. through diffusion, and/or outgassing), this may lead to EC breakdown and damaging arcing, if this is not mitigated. Due to the estimated densities of the ITER plasmas, microwaves will not be able to penetrate into the plasma in O-mode $\left(\mathbf{E}_{\mathrm{RF}} \| \mathbf{B}\right)$ at $60 \mathrm{GHz}$, so the CTS diagnostic will inject microwaves in X-mode $\left(\mathbf{E}_{\mathrm{RF}} \perp \mathbf{B}\right)$ from the low-field side.

\subsection{EC breakdown risk in the ITER CTS front-end high-power transmission line}

The EC resonances correspond to locations, where the probing frequency, $\omega_{\text {beam }}$, is equal to an integer $n$ times the local electron cyclotron frequency, $\omega_{\text {ce }}$.

$$
\omega_{\text {beam }}=2 \pi v_{\text {beam }}=n \cdot \omega_{c e}, \text { where } \omega_{c e}=\frac{e B}{\gamma m_{e}}
$$

Assuming nonrelativistic electrons in the transmission line (i.e. $\gamma=1$ ), the $60 \mathrm{GHz}$ EC resonances correspond to magnetic field strengths of $\mathrm{B}=(2.14 \mathrm{~T}) / n$. 
When calculating the local magnetic field inside the high-power transmission line fort the ITER baseline scenario $\left(\mathrm{B}_{0}=5.3 \mathrm{~T}, \mathrm{I}_{\mathrm{p}}=15 \mathrm{MA}\right)$, it is found that the $(n=1) \mathrm{EC}$ resonance is located inside EPP12 (see section 1.2).

At the EC resonance, the X-mode part of the RF electric field will be oscillating in phase with free electrons' gyromotion, and the free electrons may gain sufficient energy (from the RF field) to ionise neutral gas atoms present. If the volume-averaged ionisation rate, $v_{\text {ion, }}$, is greater than the electron diffusion rate, $v_{\text {diff }}$, the free electron density, $n_{e}$, will increase according to [5]:

$$
\begin{aligned}
& \frac{\partial n_{e}}{\partial t}=n_{e}\left(v_{\text {ion }}-v_{\text {diff }}\right) \Rightarrow \\
& n_{e}(t)=n_{e, 0} \cdot \exp \left\{\left(v_{\text {ion }}-v_{\text {diff }}\right) t\right\}
\end{aligned}
$$

If $v_{\text {ion }}>v_{\text {diff, }}$ and if the RF pulse lasts long enough for the electron density to increase up to the level of the critical electron density, where $\omega_{\text {beam }} \approx \omega_{\mathrm{pe}}=\left[n_{\mathrm{e}} e^{2} / \epsilon_{0} m_{\mathrm{e}}\right]^{1 / 2}$, an EC breakdown occurs, which may lead to strong local absorption and reflection of the gyrotron beam [6].

Both $v_{\text {ion }}$ and $v_{\text {diff }}$ depend strongly on the local strength and mutual orientation of the electromagnetic (EM) fields, as well as the neutral gas pressure, and composition. For all other parameters held constant, the ionization rate depends strongly on the local gas pressure. This means that as the neutral gas pressure increases, which is expected during long ITER plasma pulses, the system may transition from a no-EC breakdown to an EC breakdown scenario.

\subsection{ITER magnetic field inside the port plug}

For the ITER baseline scenario $\left(\mathrm{B}_{0}=5.3 \mathrm{~T}, \mathrm{I}_{\mathrm{p}}=15 \mathrm{MA}\right)$, the magnetic field strength corresponding to the $n=160 \mathrm{GHz}$ EC resonance $(\mathrm{B}=2.14 \mathrm{~T})$ is located at a tokamak major radius of $R \approx 10.4 \mathrm{~m}$ [7]. Furthermore, at the resonance, the magnetic field is found to have a significant $(\sim 26 \%)$ radial component (i.e. along the probing gyrotron beam). The magnetic field within the ITER port plugs is a complicated three dimensional quantity, which is not readily determined analytically. In this case, a 3D field map provided for a generic ITER equatorial port plug has been utilized [7]. From this, the magnetic field at the in-vessel CTS components was determined.

\section{Split Biased Waveguide}

Due to the severe engineering restrictions imposed by the nuclear ITER vacuum environment, it is not possible to implement the standard method of mitigating in-transmission line EC breakdown (i.e. controlling the local gas pressure and composition) on the ITER CTS diagnostic. Furthermore, the radial component of the ITER magnetic field is sufficiently strong that O-mode transmission will not prevent an EC breakdown at the EC resonance (see section 2.2). Instead, following a Failure Mode, Effect and Criticality Analysis (FMECA), it was decided that a longitudinally-split electrically-biased waveguide (SBWG) will be implemented to transmit the gyrotron beam through the $n=160 \mathrm{GHz}$ EC resonance in the ITER CTS diagnostic. Previously, SBWGs have successfully been implemented to avoid $60 \mathrm{GHz}$ EC breakdown in the tokamaks DITE and DIII-D [8].

The basic principle of a SBWG is that a transverse DC bias voltage (up $2 \mathrm{kV}$ in this case) is applied across the waveguide - done by splitting the waveguide along the longitudinal axis, electrically isolating the two halves, and applying an external DC voltage over the two halves (see Figure 2). This causes free electrons to diffuse to the inner waveguide wall before they have a chance to ionise any neutral atoms present. For $\mathbf{E}_{\text {bias }} \| \mathbf{B}$ - electrons are freely accelerated along the magnetic field lines on a timescale of $\tau_{\text {diff }} \approx 6 \cdot 10^{-9} \mathrm{~s}$, and for $\mathbf{E}_{\text {bias }} \perp \mathbf{B}$ - electrons drift perpendicular to the magnetic field, via the much slower $\mathbf{E}_{\text {bias }} \times \mathbf{B}$-drift $-\tau_{\text {diff }} \approx 10^{-6} \mathrm{~s}$. This 'drift 
diffusion time' must be faster than the average 'ionising-collision time' of the system in order to prevent an EC gas breakdown.
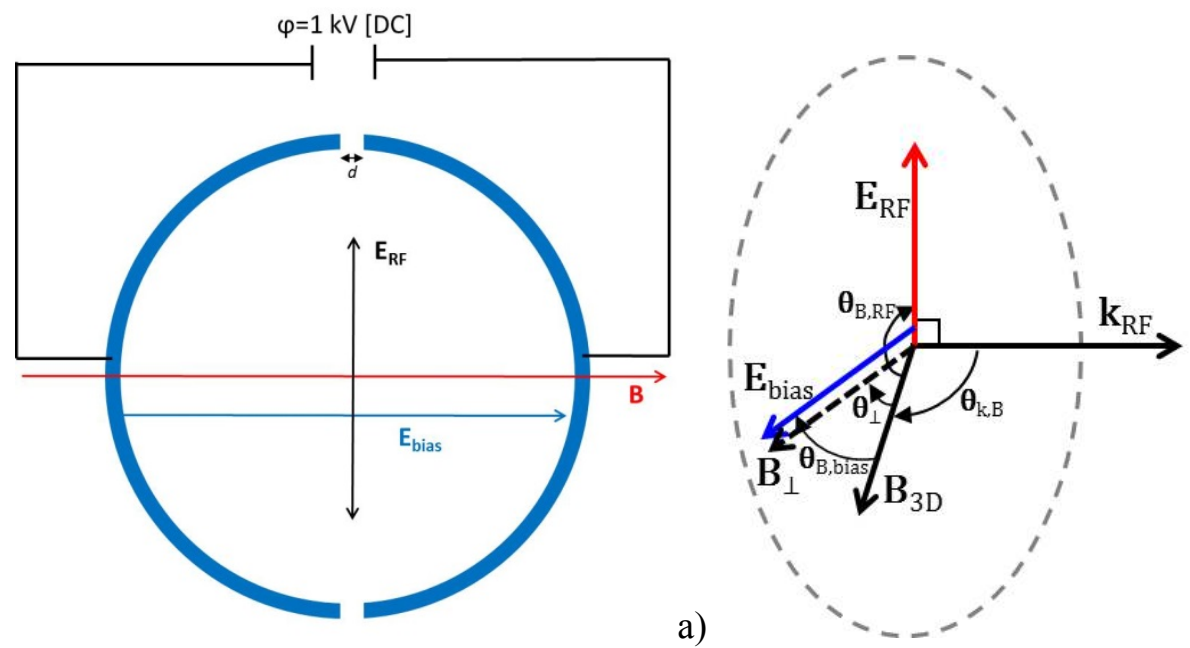

b)

Figure 2: Split biased waveguide EM field geometry: a) simple 2D transverse schematic of the field configuration inside the SBWG; and b) full 3D field configuration inside the SBWG.

\subsection{Split biased waveguide mechanical design}

The SBWG will be constructed using a sandwich design, with an inner longitudinally-split circular corrugated waveguide (WG), constructed from ITER-grade copper chromium zirconium $(\mathrm{CuCrZr})$ alloy. This will be placed inside an outer tube, constructed from ITERgrade 316LN stainless steel (SS), which provides structural stability, as well as a place to fix the clamps that fixes the SBWG to the diagnostic shielding module (DSM) and the neighbouring components. These two components will be electrically isolated from each other, by an intermediate ceramic layer constructed from aluminium oxide $\left(\mathrm{Al}_{2} \mathrm{O}_{3}\right)$, which also acts as rigid electrically-isolating spacers between the inner WG halves.

The inner WG halves will be constructed from 10 identical half-cylinders, each in electrical contact with its adjacent neighbours, and with the 2 electrical bias cables ( $1 \mathrm{kV}$ at opposite polarity) connected to the WG sections furthest away from the plasma (see Figure 3). The total length of the WG will be $1.420 \mathrm{~m}$, the inner WG diameter will be $88.9 \mathrm{~mm}$, with corrugations optimized for transmission of $60 \mathrm{GHz}$ microwaves, and a WG gap size of $d=1 \mathrm{~mm}$ (see Figure 2a). As can be determined from [7], the radial position of the EC resonance will change with changing magnetic field, so the inclusion of a longer SBWG allows the CTS diagnostic to operate at significantly different ITER magnetic field scenarios, i.e. the EC resonance will still lie within the SBWG for a central magnetic field strength of $\mathrm{B}_{0} \gtrsim 3.3 \mathrm{~T}$. For lower magnetic field scenarios, the $n=160 \mathrm{GHz}$ EC resonance will either be located in EPP12 in front of the SBWG, or within the ITER plasma.

The thermomechanical analysis of all the required system load cases is currently being performed by an external engineering company. At the time of writing this paper, the approved results of this analysis was not yet available to the CTS design team.

\subsubsection{Remote Handling \& Fixtures}

A Remote Handling (RH) assessment of the SBWG was performed, so as to address any remote handling needs in the SBWG design at an early stage. The SBWG is designed to be installed and extracted as a single RH assembly. It is fixed to its neighbouring components, and the DSM vertical blade \#1, 3, and 5, via RH compatible clamps (see Figure 3) [9]. The SBWG electrical connection will make use of a standard ITER electrical socket connector, which automatically connects/disconnects, when the SBWG is inserted into place/removed. Since the SBWG is not 
expected to be actively cooled (see section 2.1.2), no water cooling pipe cutting and welding requirements need to be taken into account.

\subsubsection{Cooling requirements}

Neutronics calculations were performed using MCNP6, so as to determine the neutronic heat load on the SBWG inner shell, and electromagnetic CST simulations were performed to estimate the Ohmic heat load from the beam on the split inner waveguide.

Based on the predicted operational temperature $\left(\sim 76^{\circ} \mathrm{C}\right)$, the deposited bulk heat load in the inner waveguide shell, due to neutrons and Ohmic losses, was determined to be on the order of $\leq 165 \mathrm{~W}$, causing a bulk temperature rise of $\leq 0.02 \mathrm{~K} / \mathrm{s}$, which produces a negligible temperature increase during a ITER baseline plasma pulse $-\tau_{\text {pulse }}=400-1000 \mathrm{~s}$. It has therefore been concluded that the SBWG will not require active cooling.

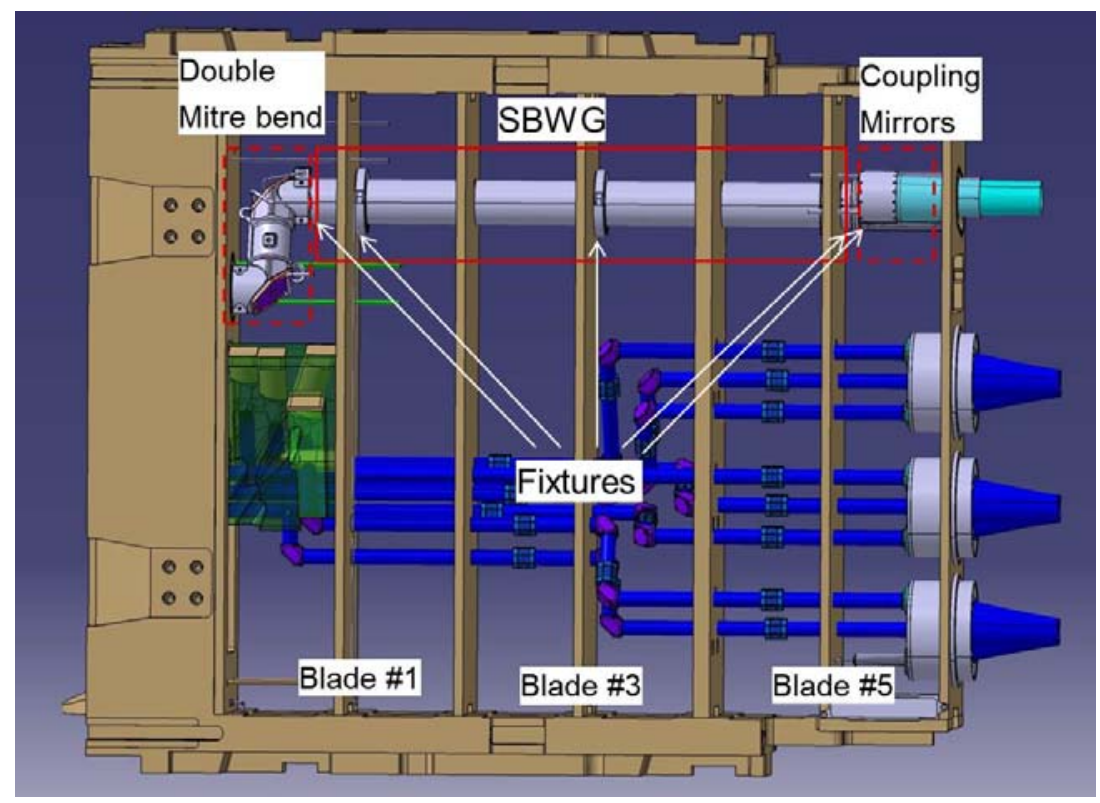

Figure 3: Overview of the SBWG integrated into the CTS system in the ITER EPP12 DSM3. Top part is the high-power launcher transmission line, while the lower part shows the low-power receiver transmission lines. The gyrotron beam (rendered in cyan) enters from the right, and exits into the plasma on the left (not shown). The bias voltage socket connector is located on the back of DSM Blade \#5.

\subsection{Monte Carlo electron modelling of EC breakdown and mitigation}

Due to the lack of an analytical theory for predicting the thresholds of EC breakdown for the relevant electromagnetic field geometry, as well as the lack of a suitable site (and budget) to perform a physical test of the functionality of the SBWG at full scale - requiring a 1+ MW 60 $\mathrm{GHz}$ gyrotron (not yet developed), a vacuum chamber, and a magnet capable of producing a homogeneous $2.14 \mathrm{~T}$ magnetic field over the diameter of the waveguide - it was decided to validate the functionality of the SBWG via computer simulations, so as to determine whether the CTS system will be at risk of EC breakdown for a given set of operational parameters.

The chosen validation method was a Monte Carlo (MC) electron particle-in-cell (PIC) simulation approach, which had previously been used to model gas discharges [10]. Based on equation 2, the MC model was modified to simulate EC breakdown and its mitigation inside the ITER CTS transmission line [11]. This model was calibrated and validated vs. the observations of SBWG functionality (EC breakdown and mitigation), when the first generation of SBWGs were tested [8]. Simulations were run until it was clear, whether the electron density was increasing or declining (i.e. whether $v_{\text {ion }}>v_{\text {diff }}$ ), which was set as the EC breakdown condition. 
The system was simulated in both 2D (EM fields shown in Figure 2a), as well as in 3D (EM fields shown in Figure 2b). In the latter case, taking the radial and axial components of the magnetic field into account. Results of 2D and 3D simulations were compared to ensure that they captured the same physics, even though the breakdown dynamics are faster in the 2D case, because the 2D field configuration is optimal for causing EC breakdown. Due to the significantly lower computational load of the 2D simulations, these were used for most of the cases and parameter studies, with the majority of the reported results coming from 2D simulations, while a few representative cases were run in full 3D.

For the ITER system (within the limits of the MC model), it was determined that EC breakdown does occur if the neutral gas pressure is sufficiently high, and no mitigation action is taken. For the 2D case, the EC breakdown pressure threshold was determined to be on the order of $p \gtrsim 0.3 \mathrm{~Pa}\left(100 \% \mathrm{H}_{2}\right)$, with the expectation that the presence of $(\mathrm{Z}>2)$ impurity gasses will lower this threshold. Depending on EM field orientation and the neutral pressure, once a sufficient RF field strength was reached, the onset time for EC breakdown was found to be 30$1000 \mathrm{~ns}-$ i.e. well within the $\sim 100 \mu$ s 'rise time' of a gyrotron pulse, and significantly faster than the shortest proposed CTS pulse length $(1 \mathrm{~ms})$. An applied transverse DC bias voltage of 2 $\mathrm{kV}$ was found to successfully mitigate EC breakdown up to a gas pressure of $p \lesssim 12 \mathrm{~Pa}(100 \%$ $\mathrm{H}_{2}$ ), with the expectation that this pressure threshold rises for the full 3D scenario.

Lastly, higher (i.e. $n \geq 2$ ) EC harmonics were not found to entail a risk of EC breakdown inside the launcher transmission line, even above the envisaged gyrotron beam power.

\section{Conclusion}

A possible ITER safety issue was identified in the ITER CTS diagnostic - the gyrotron beam encountering the fundamental EC resonance inside the in-vessel CTS launcher transmission line, which could possibly cause damaging arcing inside the ITER machine. A FMECA was performed, so as to select the best implementable EC breakdown mitigation scheme, with the result being the inclusion of a longitudinally-split electrically-biased waveguide (SBWG) in the design of the ITER CTS diagnostic system.

The risk of EC breakdown inside the in-vessel CTS launcher transmission line, as well its mitigation via a transverse DC bias voltage, has been demonstrated within the limitations of the Monte Carlo electron model (see section 2.2). The approximate low pressure threshold for onset of EC breakdown, as well as the high pressure limit of DC bias mitigation was determined (for $100 \% \mathrm{H}_{2}$ gas, and $2 \mathrm{kV}$ bias voltage). Other main findings were that $\mathrm{EC}$ breakdown is strongly dependent on the orientations of the electromagnetic fields inside the waveguide, the ambient neutral gas pressure, and the presence of gas pollutants (with $\mathrm{Z}>2$ ). Higher $\mathrm{EC}$ harmonics were not found to entail a risk of EC breakdown.

The CAD design of the SBWG is matured, with $\mathrm{RH}$ compatible fixtures, and electrical connectors. Neutronics and electromagnetic transmission simulations have been carried out on the SBWG, and have shown that bulk heating, due to neutrons and Ohmic beam loss, produces an insufficient bulk temperature rise in the inner waveguide, to necessitate active cooling, thus removing a possible safety risk to ITER, and greatly simplifying remote handling operations of the SBWG. 

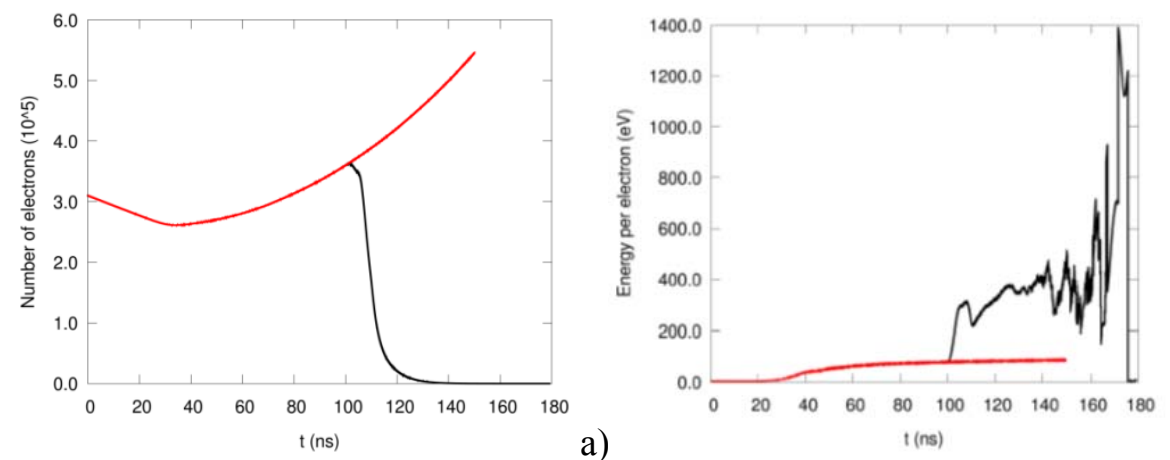

b)
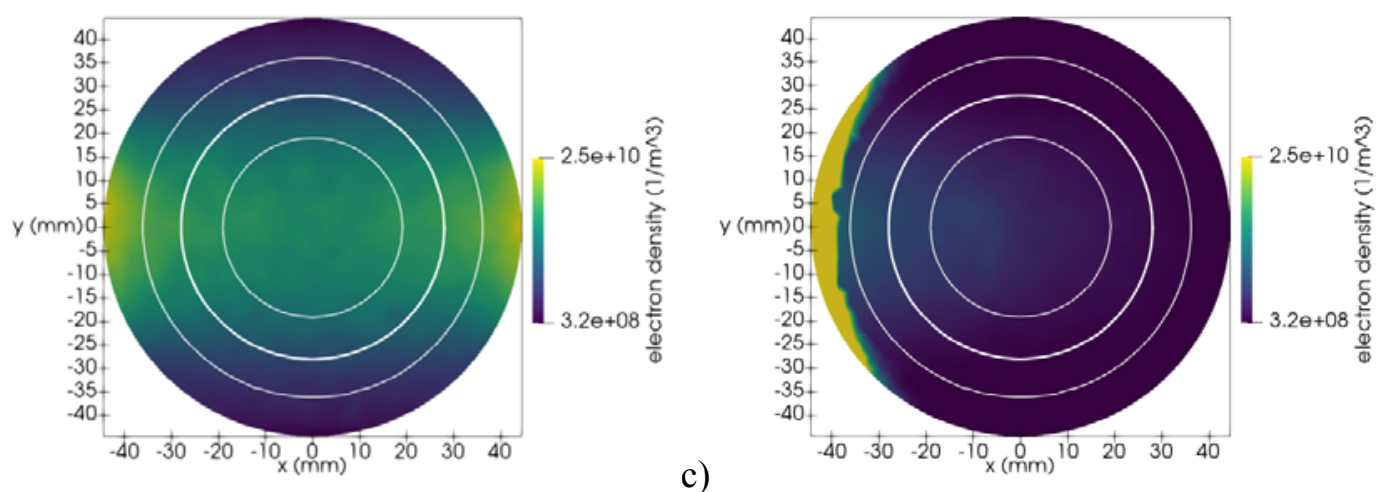

d)

Figure 4: Monte Carlo electron simulations (2D) showing the transverse cross section of the SBWG [11]. $\left(\mathbf{E}_{\mathrm{RF}} \perp \mathbf{E}_{\text {bias }} \| \mathbf{B}_{\perp}, p=1 \mathrm{~Pa} \mathrm{H}_{2}, \mathrm{E}_{0}=35 \mathrm{kV} / \mathrm{m}, \mathrm{B}_{\perp}=2.14 \mathrm{~T}, \varphi_{\text {bias }}=1 \mathrm{kV}\right)$. The contour lines indicate contours of the electric RF field strength ( $\mathrm{HE}_{11}$-mode), with the strongest field in the center of the waveguide: a) Red curve (no bias voltage) shows a more-than-linear rise, Black curve (bias voltage was turned-on at $\mathrm{t}=100 \mathrm{~ns}$ ) decreases to zero in $\Delta \mathrm{t} \approx 30 \mathrm{~ns}$. b) Black curve becomes erratic, due to poor statistics, when the electron density becomes small. c) electron density just before the bias voltage is turned-on. d) electron density $\Delta \mathrm{t}=10 \mathrm{~ns}$ after the bias voltage has been turned on - nearly all the electrons have diffused to the wall, i.e. out of the central zone in the waveguide, where the RF electric field strength, and thus electron heating is greatest.

\section{Acknowledgments}

The work leading to this publication has been funded partially by Fusion for Energy under the Framework Partnership Agreement F4E-FPA-393. This publication reflects the views only of the author, and Fusion for Energy cannot be held responsible for any use, which may be made of the information contained therein. The authors would also like to thank Charles Moeller for considerable input regarding the integration of SBWGs into existing tokamaks.

\section{References}

[1] Korsholm et al. 2019, EPJ Web Conf., 20303002

[2] M. Salewski et al. 2018, Nucl. Fusion 58096019

[3] Rasmussen et al. 2019, Plasma Phys. Control. Fusion

[4] H. Bindslev et al. 2005, Final Report EFDA Contract 04-1213-deliverable 4.1-D3.

[5] Taylor et al. 1971, Advances in Microwaves, Vol. 7 (p. 59-130)

[6] Bonatici et al. 1983, Nuclear Fusion; v. 23(9) (p. 1153-1257)

[7] ITER_D_Q2J6ME, ITER Static Flux Density EPP (coil + Plasma) v. 2.0

[8] C.P. Moeller et al. 1987, Plasma Physics and Fusion Technology (A1411), Sept 1987, p. 355-360.

[9] CTS\#D85XLF --V EP Draft, CTS ENOVIA model, v. --V

[10] Trieschmann et al. 2018, Plasma Sources Science and Technology 27 (5): 054003

[11] Trieschmann et al. 2019, Physics of Plasmas [to be submitted] 